\title{
Escitalopram-induced delayed drug rash with deranged liver function: a possible case of drug reaction with eosinophilia and systemic reaction
}

\author{
Samson YY Fong *, FHKCPsych, FHKAM (Psychiatry), YK Wing, FHKCPsych, FHKAM (Psychiatry) \\ Department of Psychiatry, Shatin Hospital, The Chinese University of Hong Kong, Shatin, Hong Kong \\ * Corresponding author: mindsmedical@yahoo.com.hk
}

Hong Kong Med J 2018;24:311-2

DOI: 10.12809/hkmj164857

\section{Introduction}

In a drug reaction with eosinophilia and systemic reaction (DRESS), patients present with a delayed onset syndrome with skin rash, haematological disturbances and systemic involvement, usually within the first 2 months of commencement of a new medication. The mortality rate is around $10 \%$. A set of diagnostic criteria has been developed by the RegiSCAR study group for potential cases of DRESS. ${ }^{1}$ A Japanese group has modified the criteria and refers to this clinical syndrome as drug-induced hypersensitivity syndrome (DISH). ${ }^{2}$ Nonetheless there is great variation in the presentation of DRESS..$^{3-5}$ Using the RegiSCAR scoring system, DRESS cases can be classified as 'no', 'possible,' 'probable' or 'definite' cases. ${ }^{4}$ Different antidepressants have been reported to cause a DRESS/DISH-like syndrome. This is the first case report of escitalopram as a possible cause of DRESS according to the RegiSCAR scoring system.

\section{Case presentation}

We report a 43-year-old Chinese male, who was in good physical health and not a hepatitis B carrier. He was first seen in the clinic of the first author in February 2014. He was a smoker but not a drinker. He had conducted liver function tests (LFTs) 2 years ago and revealed a borderline increase in alanine aminotransferase (ALT) level of $53 \mathrm{U} / \mathrm{L}$ (upper limit, $<51 \mathrm{U} / \mathrm{L}$ ) but elevated gamma-glutamyl transferase (GGT) level at $141 \mathrm{U} / \mathrm{L}$ (reference range, 10-66 U/L). No further investigation was made to determine a cause for the elevated GGT and the patient reported no physical symptoms. His chief complaints were a 1-year history of poor sleep, daytime fatigue, anxiety, poor concentration and memory, mild anhedonia, palpitations, tremor, and increased muscle tension. He reported a low mood at times but was not clinically depressed. He did not report any psychotic symptoms or suicidal ideations. He was diagnosed with generalised anxiety disorder.

The patient was treated with escitalopram $5 \mathrm{mg}$ nocte in the first week. His sleep improved but not the anxiety symptoms. On day 8 , his escitalopram was increased to $10 \mathrm{mg}$ nocte. On day 22, his anxiety symptoms improved with a transient side-effect of mild sleepiness. On day 43 , he was doing well with the treatment. On day 70, he observed an itchy, urticarial-like maculopapular rash around his waist and bilateral upper limbs. He consulted his general practitioner and was prescribed an antihistamine that did not appear to help. He attended his scheduled follow-up on day 78 . The escitalopram was reduced to $5 \mathrm{mg}$ nocte to prevent selective serotonin reuptake inhibitor discontinuation syndrome. The rash persisted and the medication was finally stopped on day 80.

On day 81 , blood tests revealed a slightly elevated white cell count of 11.69 with mainly neutrophils (7.58) but normal eosinophil count (0.28). His C-reactive protein (CRP) was elevated at $93.3 \mathrm{mg} / \mathrm{L}$ with normal C3 and C4. Elevated levels of alkaline phosphatase (ALP; $235.1 \mathrm{U} / \mathrm{L}$, normal range, 35-102 U/L), ALT (65.8 U/L, <50 U/L), and GGT (385.7 U/L, 10-66 U/L) were noted. Aspartate aminotransferase and total bilirubin were normal. On day 84, his ALP level was 247.4 U/L, GGT 348.8 $\mathrm{U} / \mathrm{L}, \mathrm{CRP}$ reduced to $15.4 \mathrm{mg} / \mathrm{L}$, and his rash began to subside. Further blood tests on day 91 revealed normalised ALT (38.3 U/L), and improved ALP (169.9 U/L) and GGT (220.1 U/L) levels. On day 92, he exhibited no drug rash nor did he report other physical symptoms. He was mentally stable with no relapse of his anxiety symptoms. On day 112, both his ALP and ALT were normalised and GGT level was reduced to $75.9 \mathrm{U} / \mathrm{L}$.

\section{Discussion}

According to the RegiSCAR scoring system, ${ }^{1}$ this is a first possible case of DRESS due to escitalopram. The patient did not present with a complete picture of DRESS (only with itchy skin rash and deranged liver function). There was no fever, lymphadenopathy, eosinophilia, or atypical lymphocytosis. His skin rash resolved spontaneously 12 days after stopping the medication. His liver enzymes had normalised within about a month of stopping medication. Anti- 
nuclear body, blood culture, hepatitis serology, serology for mycoplasma/chlamydia, and human herpes virus 6 serology were not tested because of the rapid resolution of his symptoms.

His drug rash presented with a delayed onset (10 weeks), although onset has been reported as late as 16 weeks. ${ }^{4}$ Despite an elevated baseline GGT, other baseline liver enzymes were normal. After the onset of skin rash, his liver function (ALT, ALP, GGT) became grossly deranged with a concomitant elevation of CRP, suggesting an inflammatory origin of his symptoms. It has been reported that DRESS patients, compared with those with Steven Johnson Syndrome, present with a more severe hepatocellular type of liver damage and moderateto-severe cholestatic-type liver injury. ${ }^{6}$ Other reports revealed that about $60 \%$ of subjects had abnormal LFTs, ${ }^{3,4}$ although an even higher figure of $80 \%$ has been reported in Taiwanese patients. ${ }^{5}$ Cacoub et $\mathrm{al}^{4}$ reported that among nine fatal DRESS cases, all had a skin rash and eight showed liver involvement.

Eosinophilia was not evident in our patient. While Cacoub et $\mathrm{al}^{4}$ and $\mathrm{Chen}$ et $\mathrm{al}^{5}$ reported presence of eosinophilia in $52 \%$ and $66 \%$ of patients, respectively, the figure can vary from $0 \%$ to $92 \%$ due to different precipitating medications in different case series. ${ }^{3}$ Eosinophilia may not be present in all cases of DRESS.

Clinicians should be alert for the emergence of any delayed onset skin rash after commencement of a new drug treatment. Concomitant blood tests to check for eosinophilia, deranged liver and/or renal function should be considered to exclude or diagnose DRESS.

\section{Declaration}

SYY Fong has received sponsorship for attending local and international conferences from Sanofi-Aventis Hong Kong Ltd, Pfizer Corporation Hong Kong Ltd, Otsuka Pharmaceutical (HK) Ltd, and Servier Hong Kong Ltd. YK Wing has received sponsorship from Lundbeck Export A/S, Servier Hong Kong Ltd and Celki Medical Company and was a part-time paid consultant for Renascence Therapeutics Limited.

\section{References}

1. Kardaun SH, Sidoroff A, Valeyrie-Allanore L, et al. Variability in the clinical pattern of cutaneous side-effects of drugs with systemic symptoms: does a DRESS syndrome really exist? Br J Dermatol 2007;156:609-11.

2. Shiohara $T$, Iijima $M$, Ikezawa $Z$, Hashimoto $K$. The diagnosis of a DRESS syndrome has been sufficiently established on the basis of typical clinical features and viral reactivations. Br J Dermatol 2007;156:1083-4.

3. Peyrière $\mathrm{H}$, Dereure $\mathrm{O}$, Breton $\mathrm{H}$, et al. Variability in the clinical pattern of cutaneous side-effects of drugs with systemic symptoms: does a DRESS syndrome really exist? Br J Dermatol 2006;155:422-8.

4. Cacoub P, Musette P, Descamps V, et al. The DRESS syndrome: a literature review. Am J Med 2011;124:58897.

5. Chen YC, Chiu HC, Chu CY. Drug reaction with eosinophilia and systemic symptoms: a retrospective study of 60 cases. Arch Dermatol 2010;146:1373-9.

6. Lee T, Lee YS, Yoon SY, et al. Characteristics of liver injury in drug-induced systemic hypersensitivity reactions. J Am Acad Dermatol 2013;69:407-15. 